\title{
Place des estives dans les systèmes allaitants Salers
}

\author{
Denis Cayla ${ }^{\mathrm{a} *}$, Roger Bouchy ${ }^{\mathrm{b}}$, Gilbert Liénard $^{\mathrm{c}}$ \\ ${ }^{a}$ Cemagref, Division élevage et territoires, 24, avenue des Landais, BP 50085, \\ 63172 Aubière cedex 1, France \\ ${ }^{b}$ Chambre d'agriculture du Cantal, Aurillac, France \\ c Laboratoire d'économie de l'élevage, Inra, Theix, France
}

(Reçu le 24 avril 1998 ; accepté le 8 juillet 1998)

\begin{abstract}
Role of summer mountain pastures in suckler cattle system with Salers breed. The functioning of farms which use summer mountain pastures in Cantal has been observed during 5 years. Summer mountain pastures persist mainly on altitude lands juridically bound with cattle farms. Their management differs in accordance to their production potential and they are strongly integrated in the farming system. They are specially used by suckler cattle system with Salers breed. Land costs are the greatest factor of recurrent costs of summer mountain pastures, hight recurrent costs penalize especially distant summer mountain pastures. The CAP reform is, for the moment, rather advantageous to these farms; the future of these highland pastures, essential for the tourism, is dependent on the suckler cattle systems and on the regulations that will rule these systems. $\mathcal{O}$ Elsevier/Inra
\end{abstract}

\section{Salers / summer mountain pastures / suckler cattle system / highland pastures / land costs}

Résumé - Le fonctionnement d'exploitations cantaliennes qui utilisent la transhumance a été observé sur 5 ans. Il en ressort que l'estive se maintient sur les surfaces d'altitude juridiquement rattachées aux exploitations. Leurs modes d'usage varient en fonction de leur potentiel mais elles sont fortement intégrées dans le système. Cela est surtout le fait de cheptels allaitants de race Salers. Les charges foncières représentent le facteur majeur du coût de l'estive, des coûts d'usage élevés pénalisent surtout l'estive lointaine. Les mesures liées à la réforme de la PAC sont pour l'instant plutôt favorables à ces structures ; le devenir de ces zones d'altitude, essentielles pour le tourisme, reste lié à celui de l'élevage allaitant et aux mesures qui lui seront appliquées. (C) Elsevier/Inra

Salers / transhumance / cheptels allaitants / zones d'altitude / charges foncières

* Correspondance et tirés à part

Tél. : (33) 0473440648 ; fax : (33) 04734406 98; e-mail : denis.cayla@cemagref.fr 


\section{INTRODUCTION}

La transhumance valorise de vastes territoires dans le Massif Central [1] : selon le recensement des unités pastorales de 1983, les unités de « transhumance » stricto sensu et les «montagnes » jouxtant les exploitations les plus en altitude recouvrent 80000 ha dans le Cantal et 20000 ha dans le Puy-deDôme [6]. Avec près du cinquième de sa surface fourragère elles fournissent au Cantal une contribution économique importante. Ces surfaces reçoivent des bovins si l'intérêt en est supérieur aux solutions alternatives - agrandissement et/ou intensification de l'exploitation de base, diversification - et si l'estive s'intègre bien dans les systèmes utilisateurs. Par les paysages qu'elles offrent, elles constituent un atout essentiel pour le développement actuel et futur des activités touristiques. Elles apportent enfin un support d'image pour la valorisation des productions de la région.

L'estive recouvre : i) Les montagnes rattachées au plan juridique à l'exploitation et distantes d'une dizaine à plus de $100 \mathrm{~km}$ de celle-ci ; c'est la forme essentielle de transhumance ; ii) Les mises en pension chez des particuliers ou dans des coopératives ; iii) Les sectionnaux valorisés par des ayants droit du village avec un usage souvent collectif : ce sont des territoires collectifs qui relèvent non pas de la commune mais des sections de village à l'intérieur de cette commune.

La pérennisation de l'estive suscite des interrogations du fait de son coût élevé et de certaines contraintes qu'elle entraîne pour le travail et la gestion des troupeaux. L'abandon de ces territoires d'altitude, leur retour à la friche et à la forêt, auraient des conséquences négatives pour le paysage et les activités touristiques. C'est pourquoi un observatoire de 20 exploitations a été mis en place en 1989-1990 pour 5 ans, en partenariat avec la Chambre d'Agriculture du Cantal $[2,3]$. L'objectif était de bien comprendre le fonctionnement des exploitations et les interrelations entre le mode d'exploitation de l'estive, la gestion des surfaces de base, celle du troupeau, et d'établir les résultats économiques d'ensemble.

\section{SYSTÈMES UTILISATEURS}

Parmi les utilisateurs pratiquant l'élevage allaitant en mode exclusif ou associé on distingue les élevages suivants.

- Les élevages allaitants d'altitude de grande dimension, en majorité d'estive proche et de conduite économe, qui compensent une surface de base souvent étroite par un fort recours aux estives (en moyenne 65 ha de montagne pour 76 ha de surface fourragère de base). Leur cheptel est en moyenne de 95 vaches Salers dont $80 \%$ des veaux nés sont croisés. La productivité du travail atteint 54 UGB/UTH. Le système fourrager repose sur la prairie permanente et le foin, avec des frais de la surface fourragère $(\mathrm{SF})$ réduits; le chargement de la base approche 1,2 UGB/ha de SF. En 1993/1994 ces élevages dégagent une marge par UGB Salers supérieure à $3800 \mathrm{~F}$ grâce à leur conduite économe; le revenu atteint $116000 \mathrm{~F}$ par travailleur dont trois quarts d'aides.

- Les élevages ayant un double troupeau dans le Bassin d'Aurillac et la Châtaigneraie de grande dimension, d'estive lointaine et de système intensif qui détiennent en moyenne 64 ha de montagne éloignée pour 100 ha de SF de base. Le cheptel allaitant, aussi développé que chez les élevages allaitants d'altitude - en moyenne 121 UGB Salers dans les deux cas - comporte moins de vaches car ils pratiquent l'engraissement de taurillons et de génisses. S'y ajoute un troupeau de vaches laitières spécialisées (Montbéliardes ou Holstein) de plus de 40 têtes par exploitation $(180000 \mathrm{~kg}$ de lait/an). Le système fourrager repose sur la prairie temporaire et le maïs fourrage avec ensilage et des frais fourragers élevés. Le chargement de la base atteint en moyenne 
1,5 UGB/ha de SF. En 1993/1994 ces élevages dégagent une marge/UGB Salers médiocre de $3300 \mathrm{~F}$ en raison des intrants ; mais grâce à l'importance de leur structure ils dégagent le meilleur revenu soit $170000 \mathrm{~F} /$ travailleur dont la moitié en aides.

- Les élevages ayant un double troupeau en altitude de petite dimension, d'estive proche et de conduite économe : 46 ha de base et 24 ha de montagne. Le troupeau allaitant atteint 40 UGB et moins de 30 vaches avec peu de croisement car des génisses sont vendues pour la reproduction. Le troupeau laitier spécialisé comporte un peu plus de 20 UGB lait dont 18 laitières Holstein ou Montbéliardes (100000 kg de lait/an). Le système fourrager repose sur la prairie permanente en partie ensilée (enrubannage) avec des frais modérés. Le chargement de la base est comparable à celui des allaitants d'altitude. Ces élevages obtiennent la meilleure marge par UGB Salers; le produit bovin élevé, conforté par des génisses d'élevage, s'accompagne de charges faibles. Elles dégagent ainsi une marge/UGB lait supérieure aux double troupeaux du Bassin d'Aurillac, $6300 \mathrm{~F}$ contre $4700 \mathrm{~F}$. Mais la faible dimension économique (30 UGB/ UTH) limite le revenu à $76000 \mathrm{~F} / \mathrm{UTH}$ dont la moitié en aides.

- Les élevages «mutants » de grande dimension et de système très extensif : produisant peu de lait, ils ont abandonné le double troupeau pour réduire le travail. Ces exploitations disposant d'une base de 140 ha à laquelle s'ajoute une montagne de 55 ha sont très extensives : chargement de la base de $0,8 \mathrm{UGB} /$ ha de SF. Le cheptel allaitant est important avec 132 UGB dont 94 vaches. De même dimension économique que les élevages allaitants d'altitude elles dégagent un revenu moyen identique.

Des évolutions marquées sont observées sur les 5 années en raison notamment de la réforme de la PAC.

- La surface en herbe de la base augmente de près de $10 \%$ : l'évolution est plus marquée de la $4^{\mathrm{c}}$ à la $5^{\circ}$ campagne avec l'arrivée de la prime à l'herbe.
- La surface d'herbe ensilée double avec la technique de l'enrubannage.

- L'effectif de cheptel progresse de plus de $11 \%$, mais le troupeau allaitant s'accroît de 21 UGB Salers par exploitation et la composante lait perd 8 UGB lait. En fait dans les élevages en race laitière spécialisée le troupeau laitier reste stable alors que parmi les élevages en système traditionnel Salers certains adoptent le système allaitant pur et une composante lait en race spécialisée ; ces évolutions sont accélérées par la réforme de la PAC.

- Le revenu par travailleur, médiocre au cours des quatre premières campagnes, s'améliore avec la nouvelle PAC par la conjonction de cours satisfaisants, le renforcement de la prime spéciale aux bovins mâles (PSBM) et de la prime au maintien des troupeaux de vaches allaitantes (PMTVA) avec extension de celle-ci aux troupeaux vendant moins de $120000 \mathrm{~kg}$ de lait.

\section{L'UTILISATION DES ESTIVES RESTE FORTE}

\subsection{La transhumance en montagne}

De 1990 à 1994 l'effectif bovin augmente en moyenne de 12,7 UGB par élevage dont 5,9 nourries sur des montagnes rattachées aux exploitations. Si l'effectif estivé en montagne augmente, en revanche les mises en pension et l'estive sur sectionnaux diminuent. Au total l'effectif transhumé s'accroît de 3,9 UGBa, soit le tiers de l'augmentation globale de cheptel, et la proportion moyenne d'animaux nourris à l'estive, toutes formes d'estives confondues, change peu.

$\mathrm{UGBa}=\mathrm{UGB}$ annuelles nourries à l'estive $=$ Somme des journées d'UGB à l'estive/365

Le taux d'intégration de l'estive, (UGB annuelles nourries à l'estive/UGB totales) $\times 100$, varie selon les exploitations : de moins de $10 \%$ pour une estive sur section- 
nal à titre exclusif, à un tiers des UGB annuelles pour plusieurs élevages qui transhument la quasi-totalité de leur cheptel ; les $2 / 3$ estivent 20 à $28 \%$ des UGB.

Sur les 5 ans, deux événements influent sur le taux de transhumance. En 1989/1990 la sécheresse et les dégâts de campagnols affectent l'exploitation de base et la montagne ; les éleveurs chargent moins les montagnes, réduisent la durée de présence, mettent plus d'animaux en pension et achètent beaucoup d'aliments. En 1993/1994, avec la reforme de la PAC, les éleveurs augmentent la surface de la base et le cheptel pour maximiser les primes : prime à l'herbe, PMTVA et PSBM, suppléments extensifs. Le chargement des montagnes se maintient, celui de la base agrandie baisse.

\subsection{Le troupeau Salers allaitant pourvoyeur quasi-exclusif des estives}

L'estive concerne surtout des bovins Salers : $95 \%$ de l'effectif UGBa nourri à l'estive. Les Salers allaitantes et leurs veaux représentent les trois quarts des UGBa à l'estive et les génisses Salers un cinquième ce qui est élevé. Les génisses laitières (et les chevaux) fréquentent peu ces surfaces pastorales, sauf celles gérées par les groupements pastoraux comme la Coopérative de transhumance et d'amélioration des structures agricoles (COPTASA) [8]

Un tiers du troupeau Salers allaitant des élevages avec double troupeau est nourri à l'estive $(34,2 \%$ des UGB Salers contre $24,4 \%$ en système allaitant) afin de libérer la base pour le troupeau laitier.

\subsection{Un fonctionnement de l'estive en montagne adapté à la localisation et au potentiel des montagnes}

La surface des montagnes reste identique avec une moyenne de 50 ha ; les élevages allaitants spécialisés détiennent les plus grandes montagnes jusqu'à 120 ha.

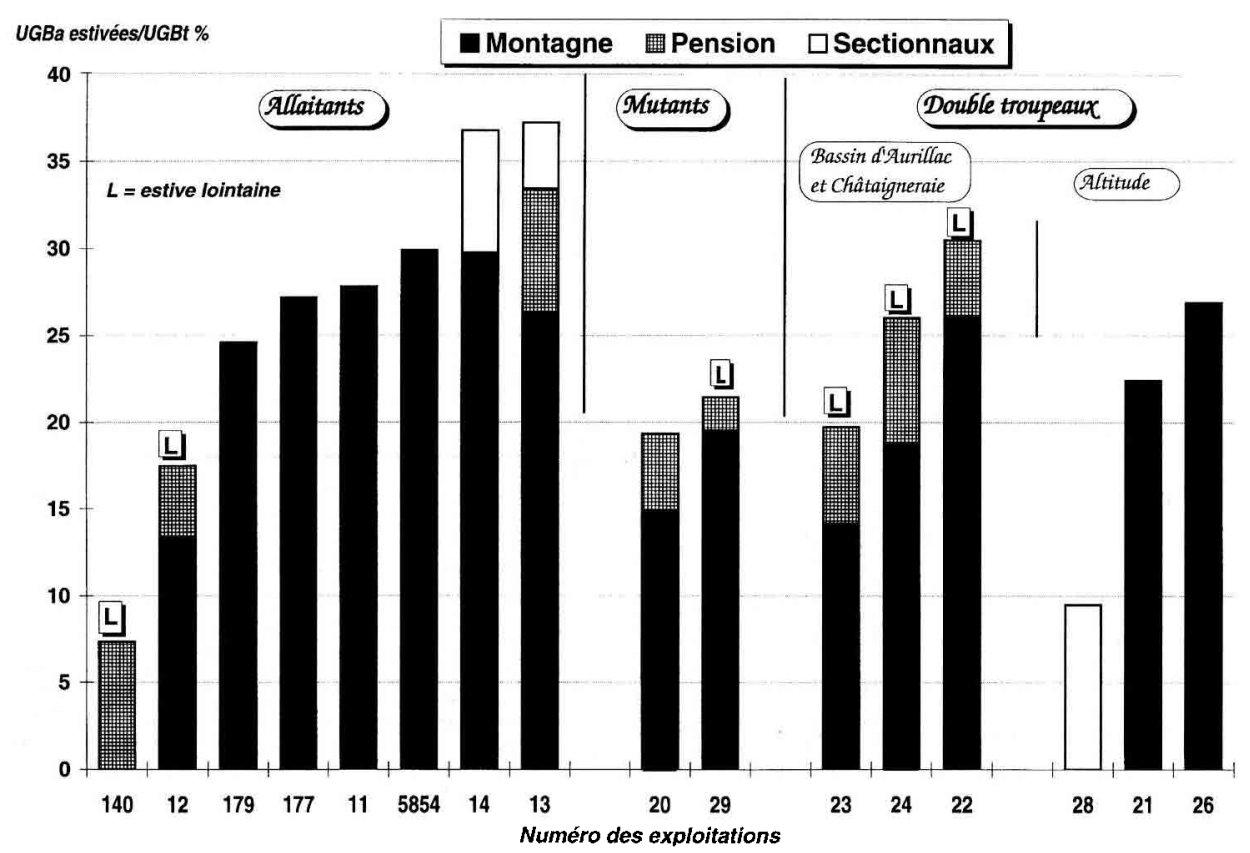

Figure 1. Taux d'intégration de l'estive selon les types d'exploitation (moyenne sur 5 ans et sur échantillon constant). 
Le chargement des montagnes pendant la période d'estive est de l'ordre de 1,25 UGB en moyenne ; le chargement annuel approche 0,5 UGBa par ha de montagne. Il varie beaucoup entre les montagnes selon :

i) l'altitude : variant de 1000 à $1450 \mathrm{~m}$ avec une forte relation négative entre le chargement et l'altitude, celle-ci influence la durée de présence des animaux et le chargement instantané ;

ii) l'apport d'engrais : facilité par l'accès des parcelles par camion il accompagne un chargement élevé ;

iii) la surface de la montagne : quand elle augmente le chargement diminue, mais les plus grands domaines d'estive se localisent en altitude ;

iv) le cloisonnement et la rotation : le chargement augmente avec le nombre de parcelles entrant dans la rotation. Mais les montagnes exploitées avec cloisonnement des parcelles se situent à plus basse altitude : les parcelles ont une surface plus restreinte, elles reçoivent de l'engrais (le plus souvent phosphocalcique) et sont pâturées sur une durée plus longue.

\subsection{Incidence de l'estive sur la conduite fourragère et les résultats zootechniques}

Les élevages avec fort taux d'intégration de l'estive se caractérisent par:

- un allongement du cycle de production ; ils produisent des mâles maigres de 18 mois croisés dits « bourrets d'herbe », des génisses de 28-32 mois engraissées à l'herbe, des génisses âgées de 18-26 mois engraissées à l'auge et à partir de 1992/1993 des taurillons finis ;

- une vente de veaux broutards plus lourds car ils reçoivent des concentrés à l'estive.

Le recours intensif à l'estive est sans effet sur la productivité en veaux.

\section{LES COÛTS D'USAGE SONT ÉLEVÉS MAIS VARIABLES SELON LES TYPES D'ESTIVE}

\subsection{Montagne}

Sur 5 ans, le coût moyen par UGB estivée ressort à $1296 \mathrm{~F}$ pour une estive proche et $2394 \mathrm{~F}$ pour une estive lointaine, soit $85 \%$ de plus. L'écart se retrouve sur la plupart des postes du coût d'usage :

Les charges foncières et sociales constituent les trois quarts du coût. Les charges foncières, héritage d'une époque où ces montagnes produisaient des fromages à forte valeur ajoutée, sont devenues très lourdes par rapport à la productivité des surfaces : les revenus cadastraux sont aussi d'autant plus élevés que les communes où se trouvent ces unités pastorales n'ont guère d'autres ressources que leur sol [7]. Les montagnes éloignées supportent des fermages élevés, en moyenne $468 \mathrm{~F} / \mathrm{ha}$. Localisées dans le Cézallier vers $1200 \mathrm{~m}$ d'altitude, mises à bail par des propriétaires différents de la base, de dimension commode (50-70 ha), d'accès facile et réputées bonnes, elles sont recherchées, y compris par les éleveurs du Nord Aveyron. Les estives proches, souvent plus en altitude (1300-1 $400 \mathrm{~m}$ ) autour des Monts du Cantal, supportent des fermages moindres : $310 \mathrm{~F} / \mathrm{ha}$ en location individualisée, $368 \mathrm{~F} / \mathrm{ha}$ en location « globalisée ». Par UGBa, les charges foncières s'élèvent en moyenne à $1035 \mathrm{~F}$ pour les estives lointaines et $681 \mathrm{~F}$ pour les proches. Elles évoluent peu sur les 5 ans, l'augmentation du chargement tendant à les réduire un peu. Les charges sociales étaient jusqu'en 1990 liées au revenu cadastral et pouvaient être estimées rigoureusement. Le régime transitoire qui a suivi, avec passage progressif de la base cadastrale à celle du revenu professionnel (avec interférence de la composition de la famille et du statut), rend l'évaluation de la part des charges sociales revenant à la montagne plus aléatoire. En moyenne sur les 5 ans, le coût est estimé à $448 \mathrm{~F} / \mathrm{UGBa}$ en estive lointaine et 
Tableau I. Estive en montagne : structure du coût suivant le lieu d'estivage ( 16 exploitations sur 5 campagnes).

\begin{tabular}{lcc}
\hline Estive & Lointaine & Proche \\
\hline UGBa estivées/exploitation & 31,9 & 26,7 \\
Charges en F/UGBa estivée : & & \\
$\quad$ Sanitaire, entretien, divers & 86 & 50 \\
Engrais & 163 & 89 \\
$\quad$ Transport & 332 & 2 \\
Surveillance & 330 & 95 \\
Fermage + foncier non bâti & 1035 & 681 \\
Charges sociales & 448 & 378 \\
Total F/UGBa & 2394 & 1296 \\
\hline
\end{tabular}

$378 \mathrm{~F} / \mathrm{UGBa}$ en estive proche avec une tendance à la réduction.

Le transport est le deuxième facteur de handicap pour les estives lointaines. En hausse constante, il atteint en 1992/1993 $332 \mathrm{~F} / \mathrm{UGB}$ annuelle ce qui représente $1 \mathrm{~F} / \mathrm{UGB} /$ jnée d'estive.

Le coût de la surveillance est plus élevé avec des estives éloignées. Pour la réduire les éleveurs recherchent des contributions locales ce qui, avec l'accroissement des effectifs, l'a en moyenne allégée de $12 \%$ sur les 5 ans.

Les dépenses d'engrais sont très variables. Elles tendent à diminuer. Seuls trois élevages, détenant les montagnes les plus chargées avec 0,60 à 0,70 UGB annuelle /ha, apportent de l'engrais tous les ans.

\subsection{Mises en pension}

La mise en pension vient souvent en complément des montagnes rattachées aux exploitations aussi concerne-t-elle des effectifs réduits. Sur cinq campagnes, la charge globale représente $2845 \mathrm{~F} / \mathrm{UGBa}$ soit $450 \mathrm{~F}$ de plus $(20 \%)$ que pour l'estive en montagne à distance. Ce coût se compose des frais de pension pour $2530 \mathrm{~F} / \mathrm{UGBa}$ (près de $90 \%$ ) et de transport pour $290 \mathrm{~F} / \mathrm{UGBa}$. Mais l'éleveur a l'avantage d'être déchargé de tout souci. Pratique de complément la mise en pension apporte de la souplesse au système, surtout pour les élevages avec des montagnes très chargées.

\subsection{Sectionnaux de village}

Ces biens sectionnaux s'étageant de $1300 \mathrm{~m}$ à $1450 \mathrm{~m}$ reçoivent des effectifs réduits, $3 \%$ des UGB estivées, et génèrent peu de charges. Mais ces terrains de qualité médiocre, peu productifs et sous-exploités supportent un chargement très inférieur à celui des montagnes et les utilisateurs se déclarent peu satisfaits. Il existe donc un réel risque d'évolution d'une partie de ces surfaces vers une lande plus ou moins arborée avec des conséquences négatives pour l'environnement.

\section{CONCLUSION}

L'estive sur des montagnes liées aux exploitations s'intègre bien dans les systèmes bovins allaitants du Cantal. Cette pratique permet de réaliser différents objectifs car l'estive peut avoir plusieurs fonctions.

- L'intensification de l'exploitation en augmentant le cheptel nourri sur la surface de base [5]. 
- La diversification du système de production, avec la séparation des territoires pâturés en été - double troupeaux où les laitières restent sur la base et les allaitantes transhument - allongement du cycle de production en allaitant, avec la conservation des mâles entre 12 et 18 mois, voire la possibilité d'un retour à la production de bœufs [4].

- La sécurisation des systèmes extensifs d'altitude en facilitant la constitution de stocks.

La réforme de la PAC de 1992 se révèle favorable à l'utilisation des estives qui peuvent bénéficier de la prime à l'herbe directement ou par l'intermédiaire des groupements pastoraux. En outre les exploitations sont confortées par le supplément extensif et par l'extension de la prime au maintien du troupeau de vaches allaitantes aux élevages mixtes (quota < à $120000 \mathrm{~kg}$ ).

Mais des questions se posent sur le devenir de l'élevage allaitant avec la future réforme de la PAC à l'horizon 2000 . Tout va dépendre à nouveau des mesures spécifiques qui seront retenues pour l'élevage extensif et les zones herbagères défavorisées. Si à l'avenir le troupeau allaitant se réduit, il en sera de même de l'estive dont le coût d'usage reste élevé du fait d'éléments peu négociables (transport, impôts fonciers et autres) avec le risque d'abandon de surfaces agricoles qui, ne trouvant plus preneur, peuvent revenir à la lande plus ou moins arborée voire à des plantations massives de résineux monospécifiques alors que leur maintien en paysage ouvert et entretenu reste essentiel.

\section{RÉFÉRENCES}

[1] Bordessoule E., Les montugnes du Massif central, espaces pastoraux et transformations du milieu rural dans les monts d'Auvergne, thèse, Université Blaise Pascal, Clermont-Ferrand, 1994.

[2] Cayla D., Marsat J.B., Bouchy R., Esteve P., Liénard G.. Pizaine M.C.. Étude technico-économique d'exploitations utilisant des estives dans le Cantal - principaux résultats après 5 années d'observations, Cemagref Riom. chambre d'agriculture du Cantal, Inra Theix, $1997.94 \mathrm{p}$

13] Cayla D., Bouchy R., Liénard G., Des estives bien intégrées constituent, malgré des coûts d'usiage élevés. un solide atout pour les exploitations allaitantes : résultats d'observations sur 5 ans dans le Cantal, 30 rencontres attour des recherches sur les ruminants, Paris, 4 ct 5 décembre 1996, pp. 77-80.

[4] Liénard G., Deudon D., Modèles de production de jeunes bovins à engraisser. L'exemple de l'Aubrac, Revue Économie Rurale 85 (1970) 84-93.

[5] Oulion G., Baud G., Quiers J.M., Genevrier E., Plantade P., Liénard G., Pizaine M.C., Étude économique de la production de jeunes bovins à engraisser en race Salcrs. I" campagne d'observation 1971-1972, 1973,70 p.

(6) SCEES, Collection de statistique agricole Recensement des unités pastorales en 1983. Étude 288, 1990, 136 p

[7] Peythieu M., Élevage extensif, charges foncières, budgets communaux et infrastructures rurales, Conseil Général du GREF (Paris), 1989. $20 \mathrm{p}$.

[8] Vilkas C., Schmitt B., Analyse des systèmes de production agricole pratiquant la transhumance, Ensaia, Pare régional des volcans d'Auvergne, $1990,38 \mathrm{p}$. 